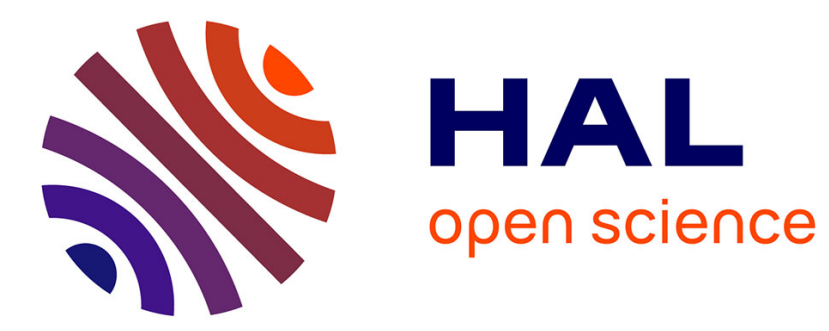

\title{
Variability of precipitating ion fluxes during the September 2017 event at Mars
}

Antoine Martinez, François Leblanc, Jean-Yves Chaufray, Ronan Modolo, Norberto Romanelli, S. Curry, J. Luhmann, R. Lillis, T. Hara, J. Mcfadden, et al.

\section{To cite this version:}

Antoine Martinez, François Leblanc, Jean-Yves Chaufray, Ronan Modolo, Norberto Romanelli, et al.. Variability of precipitating ion fluxes during the September 2017 event at Mars. Journal of Geophysical Research Space Physics, 2019, 124 (1), pp.420-432. 10.1029/2018JA026123 . insu-01971899

\section{HAL Id: insu-01971899 \\ https://hal-insu.archives-ouvertes.fr/insu-01971899}

Submitted on 11 Sep 2019

HAL is a multi-disciplinary open access archive for the deposit and dissemination of scientific research documents, whether they are published or not. The documents may come from teaching and research institutions in France or abroad, or from public or private research centers.
L'archive ouverte pluridisciplinaire HAL, est destinée au dépôt et à la diffusion de documents scientifiques de niveau recherche, publiés ou non, émanant des établissements d'enseignement et de recherche français ou étrangers, des laboratoires publics ou privés. 


\section{JGR Space Physics}

\section{RESEARCH ARTICLE 10.1029/2018JA026123 \\ Variability of Precipitating Ion Fluxes During the September 2017 Event at Mars}

Key Points:

- The solar wind dynamic pressure is the most significant during September 2017 set of space weather events

- An increase in the precipitating ion flux by more than one order during the arrival of the September interplanetary coronal mass ejection

- These results suggest that the EUV flux is not a key driver for the precipitating ion flux

Correspondence to:

A. Martinez,

antoine.martinez@latmos.ipsl.fr

Citation:

Martinez, A., Leblanc, F., Chaufray, J. Y., Modolo, R., Romanelli, N., Curry, S., et al. (2019). Variability of precipitating ion fluxes during the September 2017 event at Mars. Journal of Geophysical Research: Space Physics, 124, 420-432. https://doi.org/10.1029/ 2018JA026123

Received 26 SEP 2018 Accepted 1 JAN 2019 Accepted article online 4 JAN 2019 Published online 30 JAN 2019

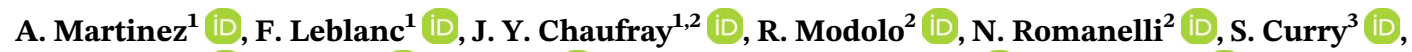 \\ J. Luhmann ${ }^{3}$ (D) R. Lillis ${ }^{3}$ (D) T. Hara ${ }^{3}$ iD, J. McFadden ${ }^{3}$, J. Halekas ${ }^{4}$ iD, F. Eparvier ${ }^{5}$ iD, D. Larson ${ }^{3}$, \\ J. Connerney ${ }^{6}$ (D) Y. J. Ma ${ }^{7}$, M. Holmström ${ }^{8}$ iD, O. Witasse9 (D) and B. Jakosky ${ }^{5}$ \\ ${ }^{1}$ LATMOS/IPSL Sorbonne Université, UVSQ, CNRS, Paris, France, ${ }^{2}$ LATMOS/IPSL, UVSQ Université Paris-Saclay, \\ Sorbonne Université, CNRS, Guyancourt, France, ${ }^{3}$ Space Science Laboratory, University of California, Berkeley, \\ California, USA, ${ }^{4}$ Department of Physics and Astronomy, University of Iowa, Iowa City, Iowa, USA, ${ }^{5}$ Laboratory for \\ Atmospheric and Space Physics, University of Colorado Boulder, Boulder, Colorado, USA, ${ }^{6}$ NASA Goddard Space Flight \\ Center, Greenbelt, Maryland, USA, ${ }^{7}$ Department of Earth Planetary and Space Sciences, UCL, Los Angeles, California, \\ USA, ${ }^{8}$ Swedish Institute of Space Physics, Kiruna, Sweden, ${ }^{9}$ ESTEC, European Space Agency, Noordwijk, Netherlands
}

Abstract In this work, we study the influence of the September 2017 solar event on the precipitating heavy ion fluxes toward Mars' atmosphere as seen by Mars Atmosphere and Volatile EvolutioN/Solar Wind Ion Analyzer, an energy and angular ion spectrometer and by Mars Atmosphere and Volatile EvolutioN/Suprathermal and Thermal Ion Composition instrument, an energy, mass, and angular ion spectrometer. After a careful reconstruction of the background induced by the Solar Energetic Particle event in the Mars Atmosphere and Volatile EvolutioN/Solar Wind Ion Analyzer spectrometer, we investigate the precipitating ion flux responses to the space weather events that took place in September 2017. This period is a unique opportunity to analyze the respective role of various possible drivers of heavy ion precipitation into Mars' atmosphere with a wide range of different space weather events occurring during the same month. This study shows an increase in the precipitation flux by more than 1 order of magnitude during the arrival of the September Interplanetary Coronal Mass Ejection compared to the average flux during quiet solar conditions. We also showed that among the possible solar drivers, the solar wind dynamic pressure is the most significant during September 2017.

\section{Introduction}

Since September 2014, the Mars Atmosphere and Volatile EvolutioN (MAVEN) mission has been dedicated to the study and the characterization of Mars' present atmospheric escape (Jakosky et al., 2015). To fulfill such objectives, MAVEN is composed of several instruments that allow us to characterize the different physical processes leading to Mars' current atmospheric escape (Lillis et al., 2015).

Atmospheric sputtering by planetary pickup ions is among the processes which may have contributed significantly to Mars' atmospheric evolution. Indeed, new ions created by the ionization of planetary neutral particles in the exosphere can be accelerated by the motional solar wind convective electric field. Depending on their location with respect to both Mars and the electric field direction, these pickup ions can impact Mars' atmosphere. Some of the energy from these ions can then be transferred by collisions to the atmospheric particles, leading to their ejection from the exosphere and the erosion of Mars' atmosphere (Johnson, 1994; Luhmann \& Kozyra, 1991).

Heavy planetary ion (which we define as having mass larger or equal to the mass of carbon atom) precipitation is the primary driver of atmospheric sputtering. The first evidence of heavy ion precipitation during quiet solar wind conditions has been reported by Leblanc et al. (2015), suggesting that atmospheric sputtering must occur almost continuously at Mars. In addition, Hara, Luhmann, Leblanc, Curry, Seki, et al. (2017) showed that the precipitating flux can be organized with respect to the orientation of the solar wind convective electric field. Lillis et al. (2015) and Edberg et al. (2009) also suggested that the precipitating flux should be dependent on other upstream solar wind conditions, such as the solar UV/EUV and the Solar Energetic Particle (SEP) flux, and the dynamic pressure. According to these articles, the EUV and SEP flux can heat and ionize the Martian upper atmosphere, potentially increasing the supply of ion that may precipitate. Moreover, the dynamic pressure compresses Mars' magnetosphere and favors the acceleration and 
precipitation of the planetary picked up ions. Also, the presence of crustal magnetic fields (e.g., Acuña et al., 1999) can also influence locally the precipitating flux as shown by Hara, Luhmann, Leblanc, Curry, Halekas, et al. (2017).

Although atmospheric sputtering is a minor contributor to the atmospheric escape today, it is thought to have been much more important four billion years ago (Chassefière \& Leblanc, 2004; Chaufray et al., 2007; Luhmann et al., 1992). The early Sun is thought to have been a much stronger emitter of both EUV and solar wind fluxes than today leading to much more intense heavy ion precipitation (Leblanc \& Johnson, 2001; Leblanc \& Johnson, 2002). Moreover, the early Sun is also expected to have been much more active, with generally more intense solar energetic events interacting with Mars much more frequently than today. In this context, the series of solar events that took place in September 2017 (Guo et al., 2018; Lee et al., 2018; Ma et al., 2018; Romanelli et al., 2018; Xu et al., 2018) is a unique opportunity to observe the effects on the heavy precipitating ion flux of an unusually high EUV flux (during the strong flare event on the 11 September as an example), but also of an Interplanetary Coronal Mass Ejection (ICME; during the 13 September encounter) and of a Corotating Interaction Region (CIR) or of a Stream Interaction Region (SIR; during the 21 September encounter).

In the present work, we describe the potential influence of the September solar events on the precipitating ion flux. In section 2, we first describe the different instruments used in this study as well as the method used to estimate and remove the background on Solar Wind Ion Analyzer (SWIA) measurements induced by the Solar Energetic Particle (SEP) events, as well as the method used to estimate the solar wind parameters. In section 3 , we present the temporal variability of the precipitating ion flux during September 2017 solar events. We compare these results with those obtained during quiet solar conditions period and discuss on the possible dependency of this precipitation with respect to solar conditions. In section 3, we conclude with a discussion and a summary of the main results of this study.

\section{Data and Method}

\subsection{Instruments and Data}

In this study, we used data from two ion spectrometers aboard the MAVEN spacecraft, namely, the Suprathermal and Thermal Ion Composition instrument (STATIC; McFadden et al., 2015) and the Solar Wind Ion Analyzer (SWIA; Halekas et al., 2015). STATIC is an energy, mass, and angular ion spectrometer operating in the range of $0.1 \mathrm{eV} / \mathrm{q}-30 \mathrm{keV} / \mathrm{q}$, with a field of view (FOV) of $360^{\circ} \times 90^{\circ}$ and a mass range from 1 to $70 \mathrm{amu}$ /q. For this study, we used exclusively the product d1 of STATIC measurements with 4-s time resolution corresponding to 32 bins in energy, 4 bins along the polar direction, 16 bins in azimuth, and 8 bins in mass. Unfortunately, during September 2017 period, STATIC was in an ionospheric mode below $500 \mathrm{~km}$ in altitude, and did not perform measurements above $650 \mathrm{eV}$. SWIA is an energy and angular ion spectrometer covering an energy range between $5 \mathrm{eV} / \mathrm{q}$ and $25 \mathrm{keV} / \mathrm{q}$ with 48 logarithmically spaced energy steps and a FOV of $360^{\circ} \times 90^{\circ}$ on 64 anodes.

In order to reconstruct the precipitating flux, we used the method developed by Leblanc et al. (2015) and Leblanc et al. (2017, 2018). We focused on a range in altitude between 200 and $350 \mathrm{~km}$, close enough to the Martian exobase so that any ion within this range of altitude with velocity at least than $75^{\circ}$ with direction respect to the local nadir direction has a very large probability to impact Mars' atmosphere. We therefore summed all measurements performed within this range of altitude if this measurement corresponded to an anode which FOV was at less than $75^{\circ}$ from the local zenith direction. In order to exclude from our analysis reconstructed precipitating flux with a poor coverage, we kept only measurements during which the average FOV covered by each instrument was more than $65 \%$ of the $75^{\circ}$ solid angle cone centered on the zenith direction.

Additionally, in order to characterize the solar wind and solar UV/EUV flux conditions (main sources of variabilities of the precipitating ion flux), this study used measurements of three other instruments: the Magnetometer (MAG) instrument (Connerney et al., 2015), the EUV monitor (Eparvier et al., 2015) which measures the solar irradiance in three bands from the soft X-ray to the extreme ultraviolet range (in three spectral bands $0.1-7,17-22$, and 121-122 nm) with a temporal resolution of $1 \mathrm{~s}$, and the Solar 
Energetic Particle (SEP) instrument (Larson et al., 2015) which measures electrons and ions with high energies (between 30 and 1,000 $\mathrm{keV}$ and 30 and 12,000 $\mathrm{keV}$, respectively).

\subsection{Methodology of Background Reconstruction for SWIA Data}

High-energy particles ( $>\sim 2-\mathrm{MeV}$ electrons and $\sim 20-\mathrm{MeV}$ protons) can induce false counts on ion and electron spectrometers (Frahm et al., 2013) equivalent to an energy-independent signal (Delory et al., 2012). During the solar event between 11 and 23 September 2017, SEPs induced a significant background in SWIA measurements for several days with a count rate peak of $1.3_{-0.1}^{+0.1} \times 10^{2}$ count/s (equivalent to an energy differential flux of $\left.3.0_{-0.5}^{+2.0} \times 10^{5} \mathrm{eV} /\left(\mathrm{cm}^{2} \mathrm{~s} \mathrm{eV} \mathrm{sr}\right)\right)$ which occurred on 12 September. In comparison, at the beginning and the end of September, the SEP background was estimated as being equal to $2.2_{-0.4}^{+0.4}$ count/s (equivalent to an energy differential flux of $6.5_{-1.5}^{+1.5} \times 10^{3} \mathrm{eV} /\left(\mathrm{cm}^{2} \mathrm{~s} \mathrm{eV} \mathrm{sr}\right)$ ). Compared to MAVEN/SWIA, MAVEN/STATIC is less sensitive to SEPs because its measurements technique is based on double coincidence (McFadden et al., 2015). Moreover, in case of intense SEP events, STATIC measurements with poor quality were flagged and excluded from our analysis.

To reconstruct the precipitating flux during the September 2017 solar events, a systematic estimate of the SEP induced background in SWIA measurements was therefore needed. As already written, the SEP-induced background is characterized by a flat spectrum of the count rate (in count/s). Because at energy larger than $5 \mathrm{keV}$, the expected ion count rate is very unlikely flat, we chose to use this energy range to estimate the SEP-induced background intensity. We defined three energy ranges: [25, 100] eV, $[100,5,000] \mathrm{eV}$, and $[5,000,25,000] \mathrm{eV}(10,26$, and 11 energy bins, respectively) and followed four steps:

1. Reconstruction of the count rate measured by each anode of SWIA on 10-min interval (144 intervals per day). For each time interval and each anode, if the count rate above $5 \mathrm{keV}$ is constant and its intensity is significantly below the count rate measured within the two other energy ranges, we considered this flux as essentially induced by SEPs. In order to determinate if the count rate spectrum is constant, we first performed a linear fit of the energy flux in the [5-25]-keV energy range. We then calculated the minimum and maximum count rate from the fitted function on the $25 \mathrm{eV}-25-\mathrm{keV}$ range. If the ratio between the maximum and minimum count rates is larger than two, we considered that the measured count rate within this energy range cannot be considered as constant. We added a second criteria to check the quality of the linear fit by calculating the root-mean-square (RMS) between the fitted function and the measurements. If the RMS was larger than 0.1 (a value empirically determined), we then considered that the quality of the fit was not good enough to be used and excluded the measurements from our analysis.

2. For each 10-min interval $t_{i}$, we averaged all the values of reconstructed background derived from the 64 anodes in a parameter $\overline{B K G}\left[t_{i}\right]$ and estimated its standard deviation.

3. The set of reconstructed average background values $40 \mathrm{~min}$ before and after MAVEN path between 200 and $350 \mathrm{~km}$ in altitude was then used to estimate the background, $B K G[t]$ for each time step $t$ within this altitude range following the equation:

$$
B K G[t]=\frac{\sum_{i=1}^{N} c_{i}[t]^{*} \overline{B K G}\left[t_{i}\right]}{\sum_{i=1}^{N} c_{i}[t]}
$$

where $t_{i}$ is the mean time of each 10-min interval, $t$ is the time of MAVEN's path between 200 and $350 \mathrm{~km}$ in altitude, $N$ is the number of reconstructed average background values (less than 9 ), and $c_{i}[t]$ is the weight of each reconstructed background $\overline{B K G}\left[t_{i}\right]$, which is equal to $\frac{1}{\left(t_{i}-t\right)^{2}}=c_{i}[t]$. This quadratic dependency was chosen to favor the time interval close to $t$.

4. For each time step $t$ within 200 and $350 \mathrm{~km}$ in altitude, we then subtracted the value of $B K G[t]$ from the SWIA data and converted the corrected count rate into particle flux.

Figure 1 displays the reconstructed background during September 2017 period. As shown, such background is very significant between 11 and 15 September, that is, during the period when MAVEN/SEP instrument measured an important flux above $100 \mathrm{keV}$ for ions and electrons. We can also note two secondary peaks on 17 and 21 September (which is visible with another data set where we displayed the median value of background per day) which correspond to two CIR/SIR encounters with SEP electron and ion fluxes significantly higher than during nominal periods. 


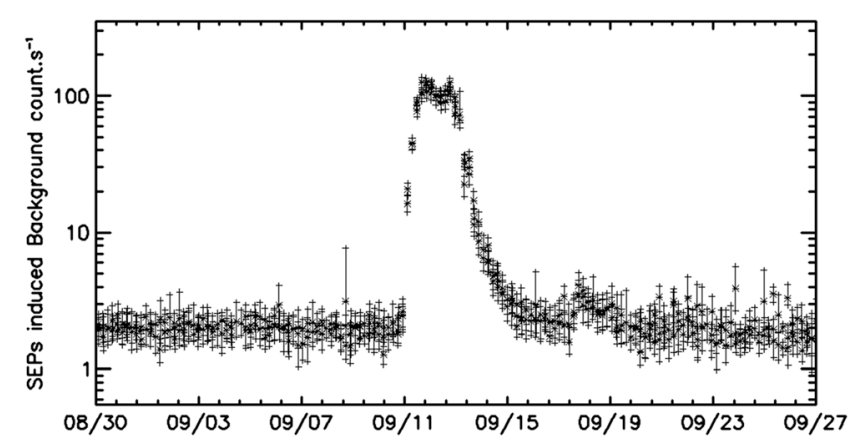

Figure 1. SWIA background estimate (with 1-sigma uncertainty) in count rate between 200 and $350 \mathrm{~km}$ in altitude.

\subsection{Reconstruction of the Solar Conditions During MAVEN Periapsis Path}

Unfortunately, during September 2017, the MAVEN orbit was entirely inside the Martian magnetosphere, and therefore, no direct measurements of the upstream solar wind conditions were available. Ma et al. (2018) therefore developed a method to derive the upstream solar wind conditions based on MAVEN measurements in the magnetosheath. These authors considered the following approaches and assumptions: the upstream IMF MSO $B_{x}$ component was assumed equal to zero and the solar wind flow to be only along the $X$ direction (that is along the Sun-Mars axis). Ma et al. (2018) simulated Mars' electromagnetic plasma environment for nominal solar wind and radiation conditions using a multispecies single MHD model. Then, they compared the simulated

plasma parameters (the solar wind speed $\vec{V}$, the interplanetary magnectic field $\vec{B}$, the solar wind density $n$, and the solar wind temperature $T$ ) along MAVEN's trajectory in the magnetosheath with MAVEN measurements. For each of these solar wind parameters, Ma et al. (2018) calculated the corresponding scaling factor defined as the ratio between the simulated variables and the measured ones, and applied these scaling factors to the upstream solar wind parameters.

This method still cannot provide an estimate of the solar wind parameters when MAVEN was in the Martian-induced magnetosphere, that is, during periods when we reconstructed the precipitating ion flux. We therefore interpolated the solar wind parameters from their reconstructed values when MAVEN was inside the magnetosheath before and after MAVEN's periapsis. We assume that the evolution of the solar wind parameters changed linearly between these two times (typically separated by $90 \mathrm{~min}$ or more):

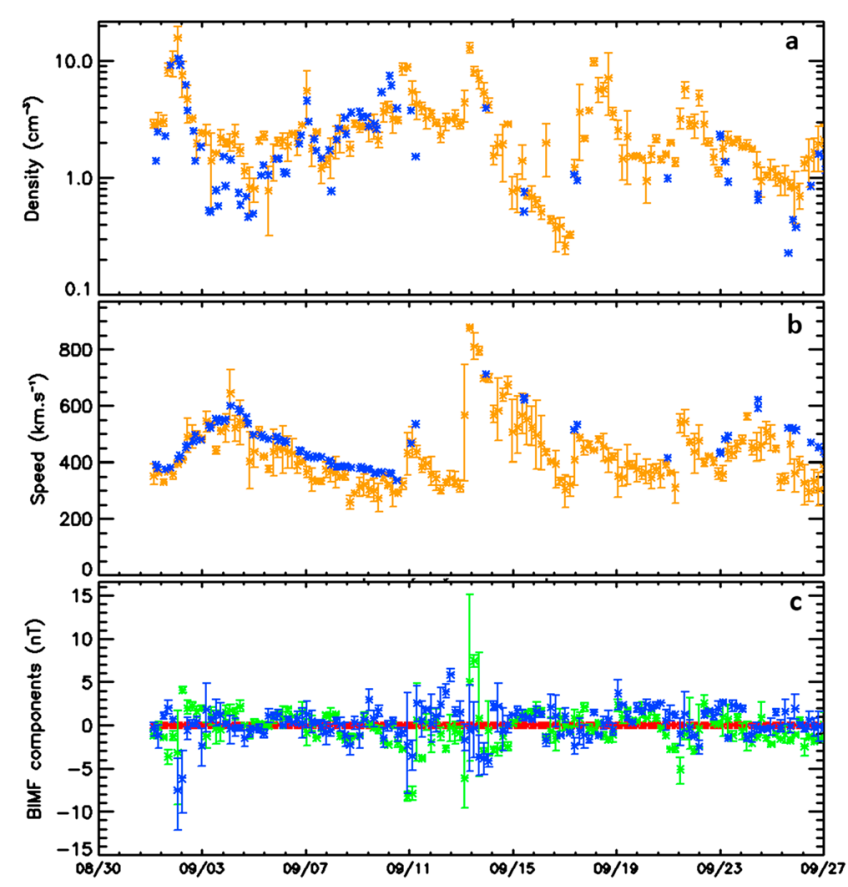

Figure 2. Comparison between the reconstructed solar wind parameters used in this paper (orange stars) and the solar wind parameters measured by MEX ASPERA3 (blue stars) during September 2017 period. (a) Solar wind density. (b) Solar wind speed. (c) Reconstructed magnetic interplanetary field (IMF) components. Red stars: $B_{x}$ (set to zero by assumption). Green stars: $B_{y}$. Blue stars: $B_{z}$.
1. Each time, MAVEN was in the magnetosheath, we made an average of the reconstructed ion density $n$, the IMF $\vec{B}=(B x, B y, B z)$, the solar wind speed $\vec{V}=(V x, V y, V z)$, and the temperature $T$, over 10-min interval. In the following, $\overline{V a r}_{0}$ is the average solar parameter at a time $t_{0}$ before the periapsis and $\overline{V a r}_{1}$ is the average solar parameters at a time $t_{1}$ after the periapsis.

2. We then linearly interpolated the reconstructed solar wind parameters from $\overline{V a r}_{0}$ and $\overline{V a r}_{1}$ to the time $t$ when we reconstructed the precipitating ion flux (as in section 2.2) using the following relation:

$$
\operatorname{Var}=\overline{\operatorname{Var}}_{0}+\left(\overline{\operatorname{Var}}_{1}-\overline{\operatorname{Var}}_{0}\right) * \frac{t-t_{0}}{t_{1}-t_{0}}
$$

In order to estimate an uncertainty for these reconstructed solar wind parameters, we considered the dispersion of the reconstructed solar wind values at $t_{0}$ and $t_{1}$. To verify the validity of our methods, we also compared these results with Mars Express measurements of the density and speed of the solar wind using ASPERA-3 (Barabash et al., 2006; Ramstad et al., 2018). In Figure 2, we displayed our interpolated solar wind parameters (in orange) and the Mars Express measured values (in blue): the solar wind density (Figure 2a) and the solar wind speed (Figure 2b). This comparison shows that our interpolation method provides a satisfying good reconstruction of these two solar wind parameters.

In the following, we will calculate the norm of the solar wind convective electric field, the dynamic solar pressure, and the coordinate of MAVEN in the Mars Solar Electric (MSE) frame when MAVEN was between 200 and $350 \mathrm{~km}$ in altitudes from the reconstructed upstream solar wind parameters $n, \vec{B}$, and $\vec{V}$. 


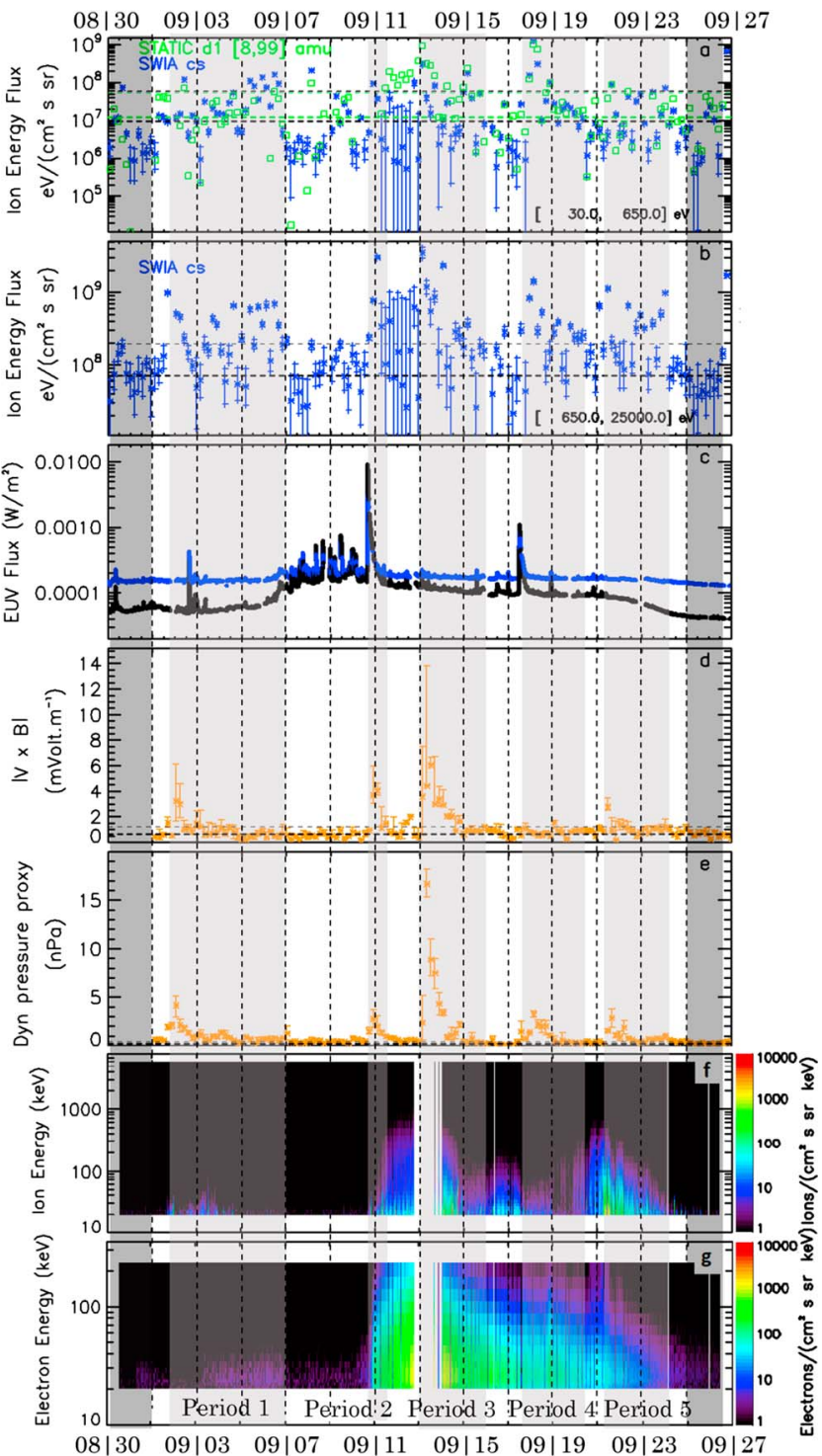

Figure 3. Measured SWIA integrated precipitating ion flux and few potential drivers of the precipitating ion flux during September 2017 period. Integrated precipitating ion flux within (a) 30- and 650-eV energy range and (b) 650-eV and 25-keV energy range. Green squares: STATIC d1 8-99-amu mass range. Blue stars: SWIA cs (coarse survey) product. The horizontal black (green) thick dashed line corresponds to the average value of SWIA (STATIC d1) precipitating ion flux during the two periods encompassing September 2017 event (in dark gray; see text for further explanation). The thin dashed horizontal line corresponds to the average value plus 3 times the corresponding standard deviation. Errors bars in (a) and (b) correspond to 1sigma deviation of the reconstructed background. (c) Values of the EUV photon flux as measured by MAVEN/EUV instrument, blue line: 17-22-nm range and black line 0.1-7-nm range. (d) Magnitude of $|\vec{V} \times \vec{B}|$ calculated from the reconstructed solar wind parameters when the penetrating ion flux was measured (see section 2.3). (e) Dynamic pressure calculated from the reconstructed solar wind parameters when the penetrating ion flux was measured (see section 2.3). Errors bars in (d) and (e) correspond to the dispersion of the corresponding interpolated value. (f) Ion flux measured by MAVEN/SEP F1 detector between $20 \mathrm{keV}$ and $70 \mathrm{MeV}$. (g) Electron flux measured by MAVEN/SEP F1 detector between 20 and $300 \mathrm{keV}$.

\section{Results}

In Figure 3a, we show the precipitating ion flux at low energy (30$650 \mathrm{eV}$ ) using both SWIA and STATIC measurements (for masses larger than $8 \mathrm{amu}$ in the case of STATIC measurement) and high energy (650 eV-25 keV) using only SWIA measurements in Figure 3b, as well as the EUV flux measured by the MAVEN/EUV instrument in Figure 3c, the $|\vec{V} \times \vec{B}|$ from the reconstructed solar wind parameters (Figure 3d), the dynamic pressure of the solar wind from the reconstructed solar wind parameters (Figure 3e), and the SEP flux in Figure 3f (ion flux) and Figure 3g (electron flux) as measured by the MAVEN/SEP instrument. The choice of the energy ranges was motivated by MAVEN/STATIC energy range during September 2017 measurements, namely, 0.1-650 eV. Moreover, MAVEN/SWIA did not perform measurements below $25 \mathrm{eV}$. We therefore define the lowenergy interval in order to compare the MAVEN/STATIC measurements with those of MAVEN/SWIA. For the high-energy range, we were limited by MAVEN/SWIA because it did not perform measurements above $25 \mathrm{keV}$.

According to Leblanc et al. (2017), the precipitating ion flux is highly dependent on the solar zenith angle, on the position of the most intense crustal magnetic field structures, and on the orientation of the convective electric field. Indeed, Hara, Luhmann, Leblanc, Curry, Seki, et al. (2017) showed that the intensity of the precipitating ion flux peaked on the hemisphere toward which the $\mathrm{E}_{\mathrm{SW}}$ field pointed (the $-\mathrm{E}_{\mathrm{SW}}$ hemisphere). In order to restrict the range of solar zenith angle covered by our sampling, we therefore chose to show only reconstructed precipitating ion flux obtained close to the MSO equator, between $-10^{\circ}$ and $15^{\circ}$ in latitude, that is, corresponding to time intervals when MAVEN is located between 200 and $350 \mathrm{~km}$ in altitude during the inbound part of its orbit. The solar zenith angle range covered by the selected set of measurements is between $49.0^{\circ}$ and $78.5^{\circ}$, the MSO longitude range between $48^{\circ}$ and $78^{\circ}$ which corresponds to the local time varying between 15.2 and $17.2 \mathrm{hr}$. We also checked that MAVEN was far from any major crustal field structures during these time intervals. We will also discuss the potential role of MAVEN MSE position.

In addition, we selected two periods before and after September 2017 solar events during which the solar wind and radiation were nominal (Nominal 0 and Nominal 6). During Nominal Period 0 and Nominal Period 6 (see Table 1), the measured solar parameters (EUV and SEP fluxes, as well as the reconstructed proxy of the solar wind parameters) suggested that the solar wind condition was "quiet" (dark gray bands in Figure 3). During these two periods, the average precipitating ion energy flux (based on 20 measurements) was $9.5 \pm 16.4 \times 10^{6} \mathrm{eV} /$ $\left(\mathrm{cm}^{2} \mathrm{~s} \mathrm{sr}\right)$ for the low-energy range and $7.2 \pm 4.2 \times 10^{7} \mathrm{eV} /\left(\mathrm{cm}^{2} \mathrm{~s} \mathrm{sr}\right)$ for the high-energy range.

Based on this nominal precipitating ion flux and its 1-sigma dispersion, we focused on periods during which the measured precipitating ion flux exceeded by more than 3-sigma (gray and green dashed line in Figures $3 \mathrm{a}$ and $3 \mathrm{~b}$ ) this nominal precipitating flux on both low- and high-energy ranges. We identified five periods in September 2017 as 
Table 1

Time Interval of Each September 2017 Period

\begin{tabular}{|c|c|c|}
\hline Period & Date Begin & Date End \\
\hline Nominal period 0 & $30 / 0802 \mathrm{hr} 09 \mathrm{~m} 09 \mathrm{~s}$ & $31 / 0822 \mathrm{hr} 34 \mathrm{~m} 45 \mathrm{~s}$ \\
\hline Period 1 & $01 / 0902 \mathrm{hr} 56 \mathrm{~m} 44 \mathrm{~s}$ & $06 / 0920 \mathrm{hr} 30 \mathrm{~m} 24 \mathrm{~s}$ \\
\hline Period 2 & $10 / 0917 \mathrm{hr} 33 \mathrm{~m} 33 \mathrm{~s}$ & $11 / 0920 \mathrm{hr} 13 \mathrm{~m} 49 \mathrm{~s}$ \\
\hline Period 3 & $13 / 0903 \mathrm{hr} 11 \mathrm{~m} 56 \mathrm{~s}$ & $15 / 0921 \mathrm{hr} 45 \mathrm{~m} 54 \mathrm{~s}$ \\
\hline Period 4 & $17 / 0900 \mathrm{hr} 18 \mathrm{~m} 49 \mathrm{~s}$ & $20 / 0921 \mathrm{hr} 34 \mathrm{~m} 15 \mathrm{~s}$ \\
\hline Period 5 & $21 / 0901 \mathrm{hr} 56 \mathrm{~m} 07 \mathrm{~s}$ & $24 / 0900 \mathrm{hr} 59 \mathrm{~m} 33 \mathrm{~s}$ \\
\hline Nominal period 6 & $25 / 0903 \mathrm{hr} 33 \mathrm{~m} 00 \mathrm{~s}$ & $26 / 0915 \mathrm{hr} 07 \mathrm{~m} 40 \mathrm{~s}$ \\
\hline
\end{tabular}

displayed in Figure 3 and in Table 1. In Figure 4, we display the average differential energy spectrum of the precipitating ion flux, measured by MAVEN/SWIA, for each selected period (in color) with the ion flux measured during the nominal period in black. The shape of the flux is very similar from one period to another one. The flux is usually maximum at low energy (below few $\mathrm{keV}$ ) then displays a plateau up to 1 to $3 \mathrm{keV}$ and then decrease down to $10 \mathrm{keV}$ (with the exception of the second and third periods where the plateau extends up to almost $10 \mathrm{keV}$ ). The typical dispersion of each period is rather large because MAVEN sampling is stable in MSO during these periods but not in Mars Solar Electric (MSE) frame (its position with respect to the $-\mathrm{E}_{\mathrm{SW}}$ hemisphere). The nominal period flux is at all energies clearly below the flux measured during these five periods.

\subsection{Period 1-Large Solar Wind Dynamic Pressure and Slow Increase of the 0.1-7-Nm EUV Flux}

In Figure 5, we display, from 1 to 7 September, the precipitating ion flux at low energy (30-650 eV) using both SWIA and STATIC measurements (for masses larger than $8 \mathrm{amu}$ ), in Figure 5a, and high energy $(650 \mathrm{eV}-25 \mathrm{keV})$ using only SWIA measurements, in Figure 5b, energy as well as the EUV flux in Figure 5c, $|\vec{V} \times \vec{B}|$ calculated from the reconstructed solar wind parameters (Figure 5d), the dynamic pressure (Figure 5e), and the SEP flux in Figure $5 f$ (electron flux) and the MSE longitude and latitude of MAVEN when MAVEN was between 200 and $350 \mathrm{~km}$ in altitudes (Figure 5g). In Figure 5, we can see that this first period corresponds to a significant increase of the proxies of the convective electric field and dynamic pressure (Figures 5d and 5e) and to a gradual increase of the EUV flux in the 0.1-7-nm range with a flare occurring at the end of 2 September (Figure 5c) with some weak SEP fluxes of electron measured during this period (Figure 5f). The fifth orbit of 1 September is not displayed because it did not fulfill the FOV threshold (see section 2.1). During this period, the average precipitating ion energy fluxes (based on 31 measurements) is equal to $3.7 \pm 4.4 \times 10^{7} \mathrm{eV} /\left(\mathrm{cm}^{2} \mathrm{~s} \mathrm{sr}\right)$ at low energy and $2.9 \pm 2.4 \times 10^{8} \mathrm{eV} /\left(\mathrm{cm}^{2} \mathrm{~s} \mathrm{sr}\right)$ at high energy, which are approximately 4 times larger than during the nominal period.

A gradual increase of the low-energy precipitating flux (Figure 5a) occurred from 5 to 6 September in an apparent de-correlation with the increase of the 0.1-7-nm flux. The high-energy flux (Figure 5b) displayed a plateau from 4 September noon up to the end of this period. It is not clearly correlated with either a plateau of the convective electric field or of the dynamic pressure. Moreover, the end of 2 and 7 September correspond to very low precipitating ion flux, whereas the 0.1-7-nm EUV flux increased significantly. According to Figure 5g, these low fluxes cannot be explained by the MAVEN MSE position. According to Edberg et al. (2009), an increase of the EUV flux might lead to push the Induced Magnetospheric Boundary (IMB) away from the planet. The simultaneous decrease of the dynamic pressure and increase

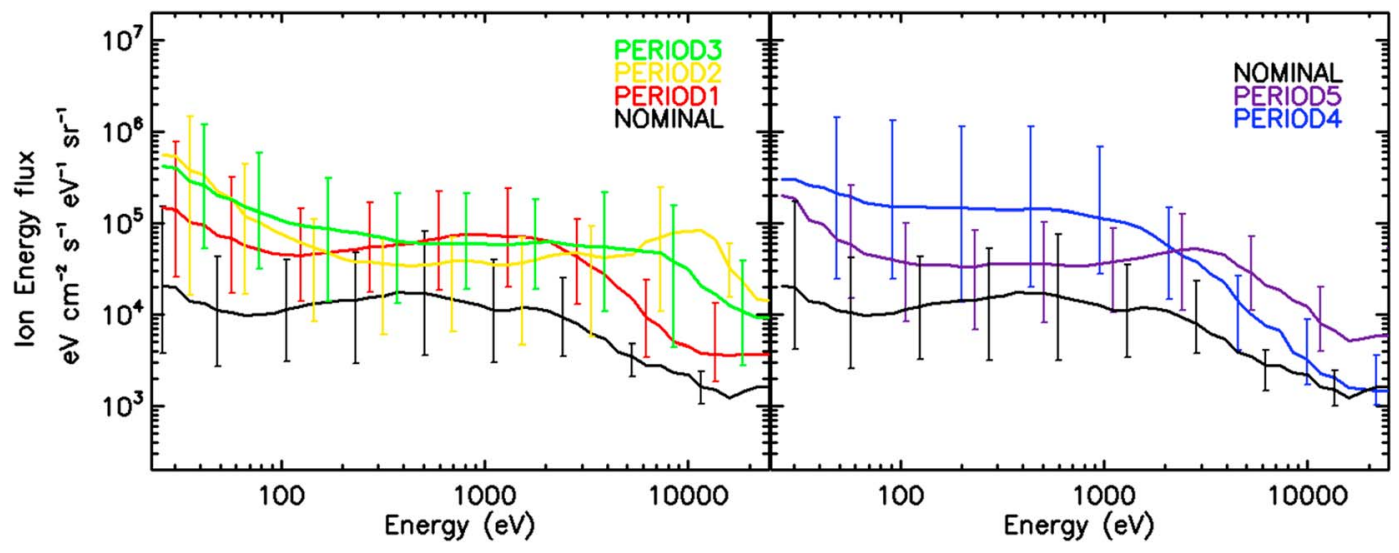

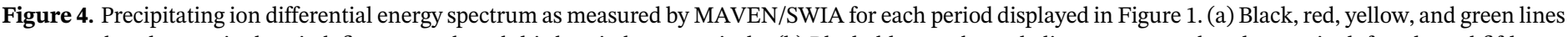

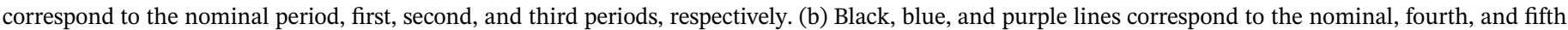
periods, respectively. Error bars are for 1-sigma dispersion calculated from the precipitating ion energy fluxes measured during each period. 


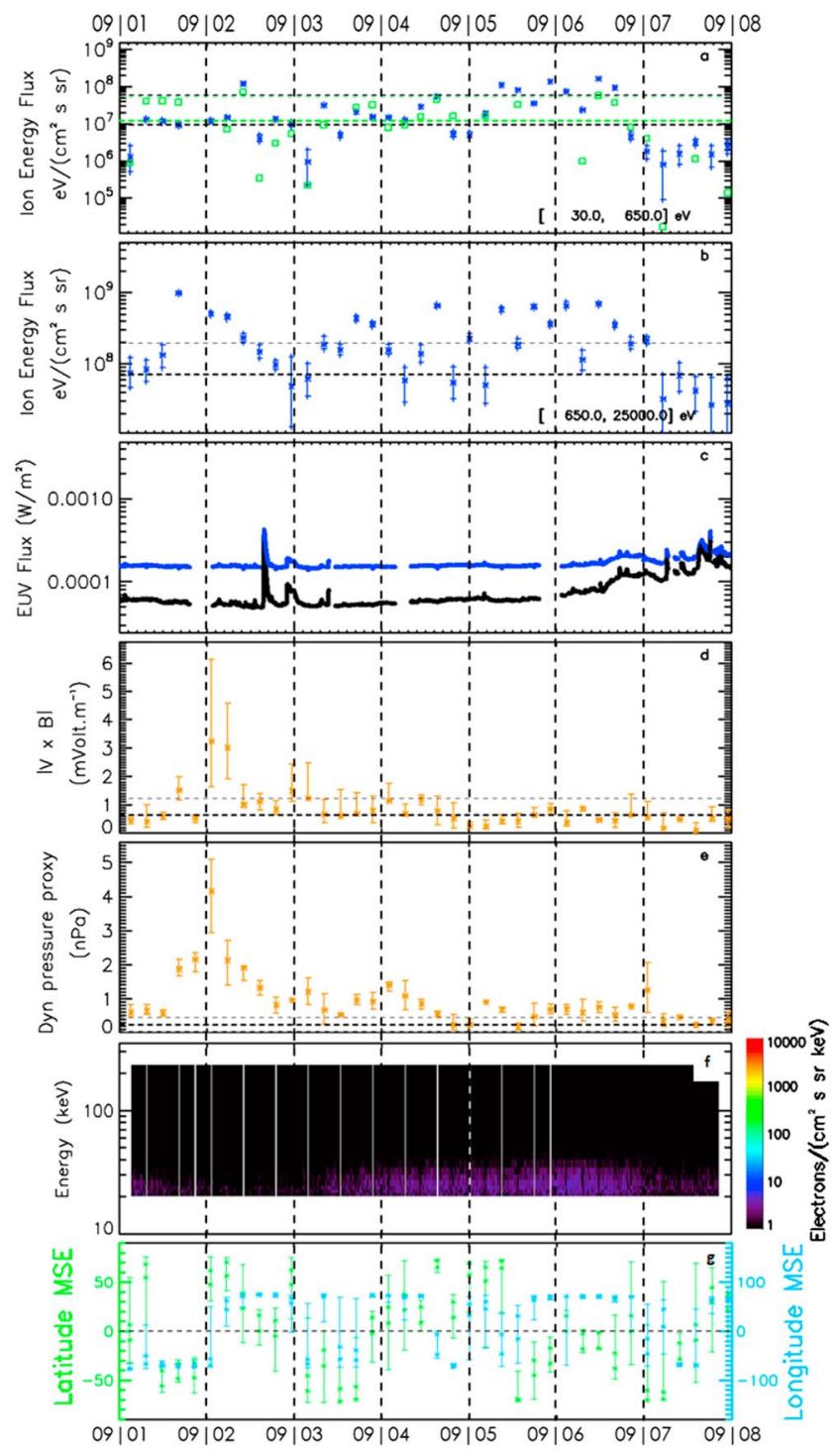

Figure 5. Measured SWIA integrated precipitating ion flux and few potential drivers of the precipitating ion flux between 1 and 7 September (period 1). Integrated precipitating ion flux within (a) 30- and 650-eV energy range and (b) 650-eV and 25-keV energy range. Green squares: STATIC d1 8-99-amu mass range. Blue stars: SWIA cs (coarse survey) product. The horizontal black (green) thick dashed line corresponds to the average value of SWIA (STATIC d1) precipitating ion flux during the two periods encompassing September 2017 event (see text for further explanation). The thin dashed horizontal line corresponds to the average value plus 3 times the corresponding standard deviation. Errors bars in (a) and (b) correspond to 1-sigma deviation of the reconstructed background. (c) Values of the EUV photon flux as measured by MAVEN/EUV instrument, blue line: 17-22nm range and black line 0.1-7-nm range. (d) Magnitude of $|\vec{V} \times \vec{B}|$ calculated from the reconstructed solar wind parameters when the penetrating ion flux was measured (see section 2.3). (e) Dynamic pressure calculated from the reconstructed solar wind parameters when the penetrating ion flux was measured. Errors bars in (d) and (e) correspond to the dispersion of the corresponding interpolated value (see section 2.3). (f) Electron flux measured by MAVEN/SEP F1 detector between 20 and $300 \mathrm{keV}$. (g) Estimated MSE latitude (green) and longitude (blue) position of MAVEN when the penetrating ion flux was measured. Errors bars on (g) correspond to the dispersion of the corresponding interpolated value (see section 2.3). of the EUV flux from 6 September could induce an IMB further from the planet. Such change of position of the IMB would push away from the atmosphere the region where picked up ions are accelerated leading potentially to a decrease of the precipitating ion flux also consistent with the much lower flux at high energy. What seems to control the precipitating flux on 1 and 2 September is actually the association of several solar drivers, the peak of precipitating occurring when both proxies of the convective electric field and dynamic pressure reached significant large intensity. However, the very large high energy fluxes measured between 5 and 7 September are difficult to associate with any significant variation of the solar wind parameters displayed in Figure 5 beside a relatively moderate increase of the solar wind dynamic pressure and of the SEP electron flux.

\subsection{Period 2-X-Flare, SEP Event, and Increase of the Solar Wind Convective Electric Field and Dynamic Pressure}

The second period of September 2017 that shows a significant increase of the precipitating ion flux corresponds to a combination of a X-flare occurring on 10 September at $15 \mathrm{hr} 45$ (Figure 3c), of an ion and electron SEP event starting on 10 September at 19 hr00 (Figures $3 \mathrm{f}$ and $3 \mathrm{~g}$ ) and a significant increase of the solar wind convective electric field and dynamic pressure as suggested by the reconstructed proxy in Figures 3d and 3e. As explained in section 1, the association between a peak in EUV flux leading to an increase of ionization with a peak of convective electric field and dynamic pressure are expected to be very favorable to the production, acceleration, and precipitation of planetary ions. The increase of the precipitating ion flux at low energies seems to follow closely the X-flare occurrence, whereas the high energy flux increases significantly few hours later when the solar electric field increased. In fact, the SEP ion flux increase displayed in Figure $3 \mathrm{f}$ seems correlated with the late increase of the precipitating ion flux at high energy (that is, slightly delayed with respect to the X-flare), suggesting that the ions observed by the SEP instrument are partly planetary heavy picked up ion accelerated during this solar event. The average differential energy flux during the second period is displayed in yellow in Figure 4 . We can clearly see that the precipitating ion flux displays a peak around $12-15 \mathrm{keV}$, strongly suggesting that the measured precipitating ion flux is mainly composed of heavy ions and most probably of $\mathrm{O}^{+}$ (Rahmati et al., 2015, 2017). The sharp increase of the electron SEP flux at the beginning of period 2 might have also contributed to increase the ionization rate and by consequence the precipitating flux. However, SEP electron flux appears not to be a key driver despite its continuous increase up to 13 September, because the high-energy precipitating flux seems not to follow the SEP electron flux variation (contrary to the low-energy precipitating flux). Actually, the high-energy precipitating flux seems rather to follow the variation of the electric field and dynamic pressure displayed in Figures 3d and 3e. According to Lee et al. (2018), such brief but significant increase of the electric field (Figure 3d) and dynamic pressure (Figure 3e) on 11 September is a consequence of a SIR encounter. The evolution of the precipitating ion flux is consistent at low energy with the simulations of Wang et al. (2015), which simulated the spectra of the precipitating $\mathrm{O}^{+}$ion flux for maximum and minimum solar 

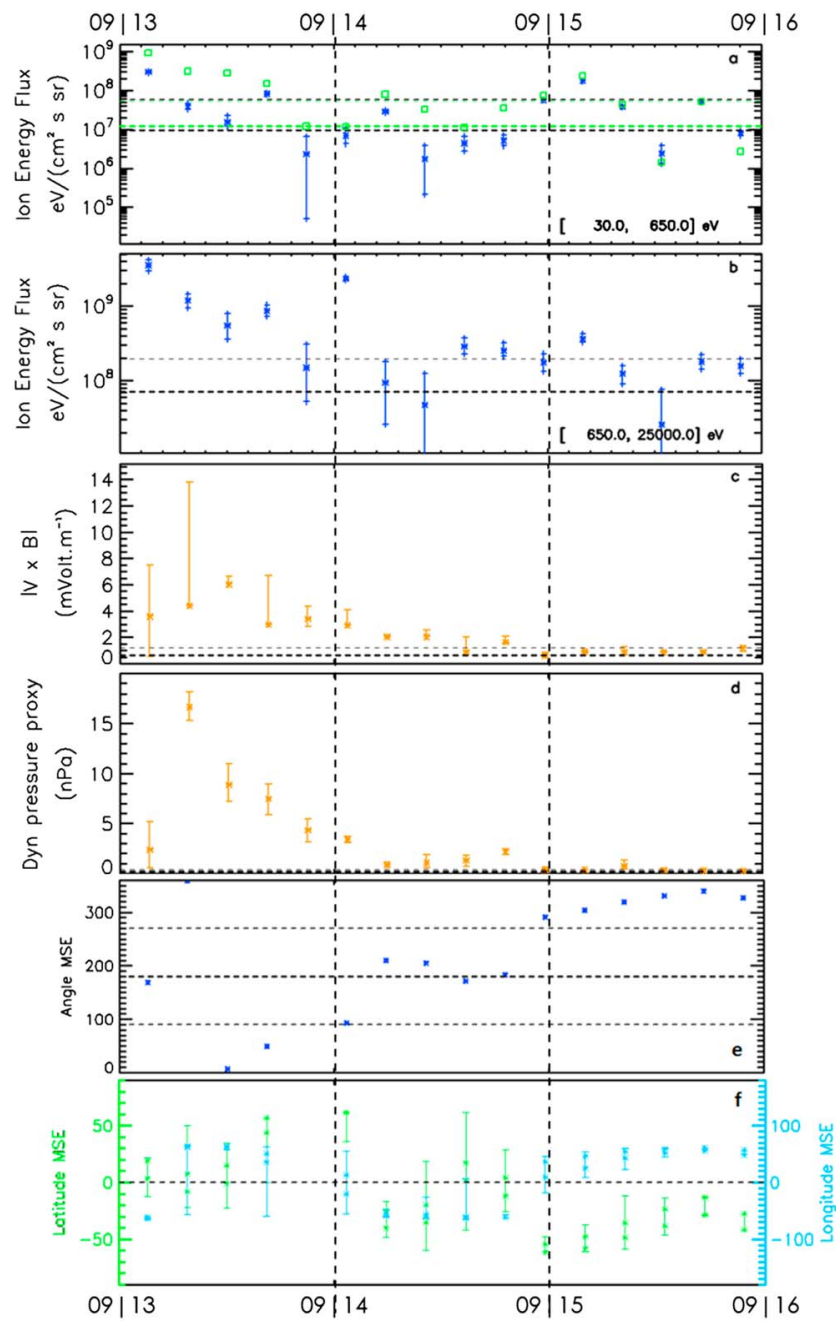

Figure 6. Measured SWIA integrated precipitating ion flux and few potential drivers of the precipitating ion flux between 13 and 15 September (period 3). Integrated precipitating ion flux within (a) $30-$ and $650-\mathrm{eV}$ energy range and (b) 650-eV and 25-keV energy range. Green squares: STATIC d1 8-99-amu mass range. Blue stars: SWIA cs (coarse survey) product. The horizontal black (green) thick dashed line corresponds to the average value of SWIA (STATIC d1) precipitating ion flux during the two periods encompassing September 2017 event (see text for further explanation). The thin dashed horizontal line corresponds to the average value plus 3 times the corresponding standard deviation. Errors bars in (a) and (b) correspond to 1-sigma deviation of the reconstructed background. (c) Magnitude of $|\vec{V} \times \vec{B}|$ calculated from the reconstructed solar wind parameters when the penetrating ion flux was measured (see section 2.3). (d) Dynamic pressure calculated from the reconstructed solar wind parameters when the penetrating ion flux was measured (see section 2.3). (e) The MSE angle of MAVEN when the precipitating ion flux was measured. The horizontal black lines correspond to $90^{\circ}, 180^{\circ}$, and $270^{\circ}$ values. (f) Estimated MSE latitude (green) and longitude (blue) position of MAVEN when the penetrating ion flux was measured. Errors bars in (c), (d), and (f) correspond to the dispersion of the corresponding interpolated value (see section 2.3 ). conditions as well as for quiet and active conditions. Our nominal period can be compared to their dayside modeling of the minimum solar activity. Differences between our measured energy distribution and the ones simulated by Wang et al. (2015) at high energy and for the case of a ICME are probably partially due to our limited spatial sampling. Fang et al. (2013) showed that the precipitating flux could vary by an order of magnitude depending on the position in MSO.

\subsection{Period 3-ICME Arrival, Large SEP Fluxes, and Slow Decay of the EUV Flux}

Period 3 is characterized by the ICME arrival on 13 September at $02 \mathrm{hr} 50$ (according to MSL/RAD; Guo et al., 2018), which was responsible for the strong increase in the solar wind dynamic pressure, shown in Figures 3e and 6d. This increase took place from the end of 12 up to 14 September, leading to a significant compression of Mars' magnetosphere and favoring the acceleration and precipitation of planetary picked up ions (Romanelli et al., 2018), a phenomenon also observed during Mars encounter with an ICME on 8 March 2015 (Curry et al., 2015). The responses of the Martian magnetosphere to the ICME on 13 September 2017 are described by Romanelli et al. (2018) using MAVEN data and the multispecies Latmos Hybrid Simulation model (LatHyS) described in Modolo et al. (2016). This study was carried out by analyzing the ICME passage by means of three stationary simulations (before the passage, during the impact, after the impact) and comparing the predicted responses and evolution of the magnetosphere with MAVEN magnetic field and plasma data along its trajectory. In Romanelli et al. (2018), the ICME encounter was characterized by an increase in dynamic pressure causing a compression of the magnetosheath and of the bow shock, followed few hours later by a change in direction of the IMF, also suggested in Ma et al. (2018).

In Figure 6, we provided a zoom into period 3 with the low-energy range precipitating flux (Figure 6a) and the high-energy flux (Figure 6b), with the proxy of the convective electric field when the penetrating ion flux was measured (Figure 6c), the proxy of the dynamic pressure when the penetrating flux was measured (Figure 6d), the MSE angle (Figure 6e), and the MSE longitude and latitude position of MAVEN when the penetrating flux was measured (Figure 6f). The MSE angle corresponds to the counterclockwise angle between the vector formed by the latitude and the longitude in MSE of MAVEN during its measurements of the precipitating ion flux and the East MSE direction (MSE longitude equal to $+180^{\circ}$ and latitude equal to $0^{\circ}$ ). According to Hara, Luhmann, Leblanc, Curry, Seki, et al. (2017), the precipitating ion flux should reach a maximum around a MSE angle of $270^{\circ}$ (ax -E). Indeed, planetary picked up ions should precipitate toward the southern hemisphere $(-\mathrm{E})$ because of the acceleration of the electric field of convection toward this hemisphere. Extreme solar events can significantly increase precipitation flux in the northern hemisphere. It is the case for this period where the solar is disturbed by the ICME impact.

As shown in Figure 6, during the fifth orbit after the arrival of the ICME (on 13 September at $21 \mathrm{hr}$ ), the measured precipitating ion flux at low energy is particularly small with respect to the nominal. The very significant difference between STATIC and SWIA fluxes at the beginning of the period can be explained by the 


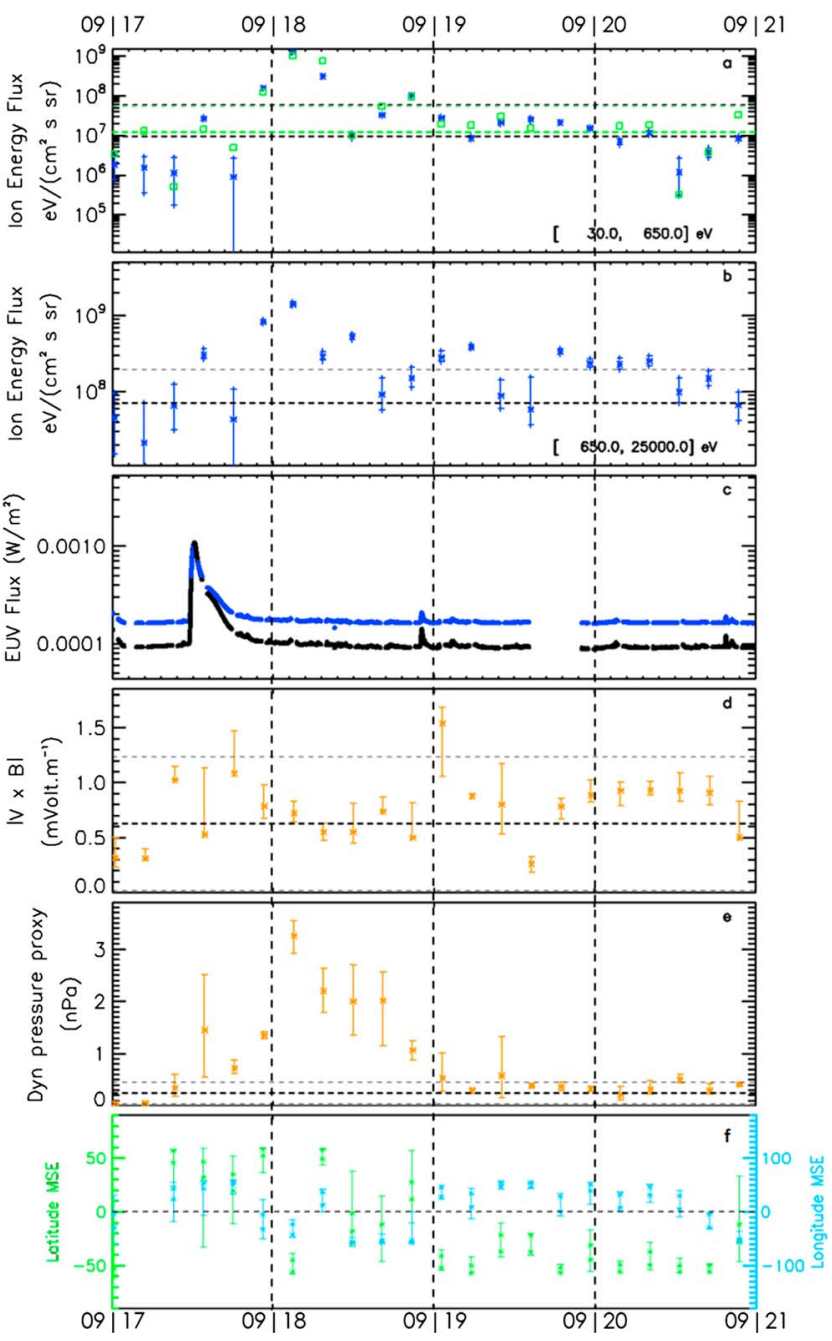

Figure 7. Measured SWIA integrated precipitating ion flux and few potential drivers of the precipitating ion flux between 17 and 20 September (period 4). Integrated precipitating ion flux within (a) 30- and 650-eV energy range and (b) 650-eV and 25-keV energy range. Green squares: STATIC d1 8-99-amu mass range. Blue stars: SWIA cs (coarse survey) product. The horizontal black (green) thick dashed line corresponds to the average value of SWIA (STATIC d1) precipitating ion flux during the two periods encompassing September 2017 event (see text for further explanation). The thin dashed horizontal line corresponds to the average value plus 3 times the corresponding standard deviation. Errors bars in (a) and (b) correspond to 1sigma deviation of the reconstructed background. (c) Values of the EUV photon flux as measured by MAVEN/EUV instrument, blue line: 17-22-nm range and black line 0.1-7-nm range. (d) Magnitude of $\vec{V} \times \vec{B}$ calculated from the reconstructed solar wind parameters when the penetrating ion flux was measured (see section 2.3). (e) Dynamic pressure calculated from the reconstructed solar wind parameters when the penetrating ion flux was measured (see section 2.3). (f) Estimated MSE latitude (green) and longitude (blue) position of MAVEN when the penetrating ion flux was measured. Errors bars in (d)-(f) correspond to the dispersion of the corresponding interpolated value (see section 2.3). important SEP flux inducing a significant background on SWIA data. The remaining SWIA flux is therefore not known with a signal/noise ratio larger than 1 . We can see a relationship between the increase of the dynamic pressure and the sharp increase of the precipitating ion flux at low energy (Figure 6a) and in SWIA measurements at high energy (Figure 6b). The average precipitating energy fluxes (based on 16 measurements, respectively) at low energy are $5.1 \pm 7.9 \times 10^{7} \mathrm{eV} /\left(\mathrm{cm}^{2} \mathrm{~s} \mathrm{sr}\right)$ and at high energy $6.5 \pm 9.7 \times 10^{8} \mathrm{eV} /\left(\mathrm{cm}^{2} \mathrm{~s} \mathrm{sr}\right)$, which are approximately 5 and 9 times more important than during the nominal period.

The precipitating ion flux decreases just after the arrival of the ICME but remains significantly larger during more than $48 \mathrm{hr}$ after this time. The minimum of the high energetic flux at noon on 13 September seems to be associated to a significant change of MAVEN in the MSE frame as illustrated in Figure 6e. As displayed in Figure 4, the average differential energy flux during this period displayed a peak around $10 \mathrm{keV}$ that is probably due to planetary picked up ions (Rahmati et al., 2015). As suggested by STATIC d1 measurements at low energy (Figure 6a), the passage of the ICME also causes a strong increase of the planetary heavy pickup ions at low-energy range. After the middle of 14 September, the measured precipitating ion fluxes at low and high energies do not display significant intensity with the exception of the beginning of 15 September which corresponds to a minimum in MSE latitude (Figure 6f).

\subsection{Period 4-Flare Event, Large SEP Flux, and Large Solar Wind Dynamic Pressure}

Figure 7 is a zoom of period 4 displaying the precipitating ion flux at low(Figure 7a) and high-energy (Figure $7 b$ ) with the values of the EUV photon flux as measured by MAVEN/EUV instrument (Figure 7c), the proxy of the convective electric field when the penetrating ion flux was measured (Figure 7d), the reconstructed dynamic pressure when the penetrating ion flux was measured (Figure 7e), and the MSE longitude and latitude position of MAVEN when the penetrating ion flux was measured (Figure 7f). Period 4 is characterized by the occurrence of a M-flare 17 September (Figure 7c). During this period, the SEP flux was decreasing with respect to periods 2 and 3 (Figures $3 f$ and $3 g$ ) but remained significantly higher than the SEP flux during the nominal period. We also notice a moderate increase of the dynamic pressure at the beginning of 18 September (Figure 7e) with respect to the third period but still significantly higher than the dynamic pressure during the nominal period.

The average precipitating ion energy fluxes (based on 22 measurements) at low energy are $9.2 \pm 26.3 \times 10^{7} \mathrm{eV} /\left(\mathrm{cm}^{2} \mathrm{~s} \mathrm{sr}\right)$ and at high energy $2.7 \pm 3.2 \times 10^{8} \mathrm{eV} /\left(\mathrm{cm}^{2} \mathrm{~s} \mathrm{sr}\right)$, which are almost 10 and 4 times larger than the fluxes measured during the nominal period. As shown in Figure 4, this is the largest average precipitating ion flux of the five September periods, in particular at low energy. Actually, this large flux at low energy is dominated by 18 September flux and is associated with the peak of solar dynamic pressure and the decay of the flare. As displayed in Figure 4, the average differential energy flux during this period displayed a peak around $1-3 \mathrm{keV}$ that is probably due to planetary picked up ions, as suggested by STATIC d1 measurements at low energy (Figure 7a).

From the fifth orbit of the period, there is a gradual increase in dynamic pressure until the beginning of 18 September. The dynamic pressure then decreased until 19 September. During this decrease, the evolution of the precipitating ion flux seems to follow this trend, but is also influenced, at high energy, by the variation of 


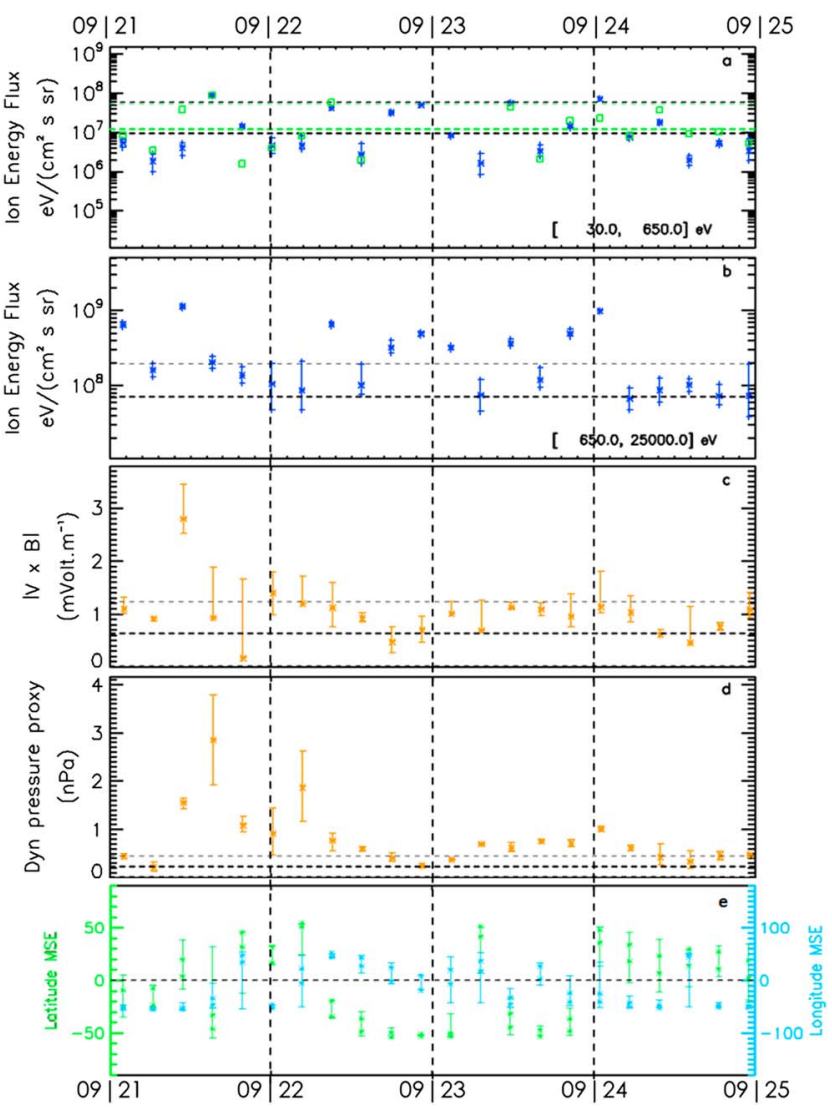

Figure 8. Measured SWIA integrated precipitating ion flux and few potential drivers of the precipitating ion flux between 21 and 24 September (period 5). Integrated precipitating ion flux within (a) 30- and 650-eV energy range and (b) 650-eV and 25-keV energy range. Green squares: STATIC d1 8-99-amu mass range. Blue stars: SWIA cs (coarse survey) product. The horizontal black (green) thick dashed line corresponds to the average value of SWIA (STATIC d1) precipitating ion flux during the two periods encompassing September 2017 event (see text for further explanation). The thin dashed horizontal line corresponds to the average value plus 3 times the corresponding standard deviation. Errors bars in (a) and (b) correspond to 1 sigma deviation of the reconstructed background. (c) Magnitude of $|\vec{V} \times \vec{B}|$ calculated from the reconstructed solar wind parameters when the penetrating ion flux was measured (see section 2.3). (d) Dynamic pressure calculated from the reconstructed solar wind parameters when the penetrating ion flux was measured (see section 2.3). (e) Estimated MSE latitude (green) and longitude (blue) position of MAVEN when the penetrating ion flux was measured. Errors bars in (c)-(e) correspond to the dispersion of the corresponding interpolated value (see section 2.3). the intensity of the electric field. We found a linear correlation coefficient of 0.69 on 21 SWIA measurements and 0.72 on 19 STATIC d1 measurements at low energy between the precipitating ion flux and the dynamic pressure.

\subsection{Period 5-Large Electric Field Intensity and Solar Wind Dynamic Pressure}

The last significant increase of the precipitating ion flux during September 2017 occurred from 21 up to 24 September with an average flux (based on 17 measurements) of $2.4 \pm 2.8 \times 10^{7} \mathrm{eV} /\left(\mathrm{cm}^{2} \mathrm{~s} \mathrm{sr}\right)$ for the low energy range and $4.0 \pm 3.4 \times 10^{8} \mathrm{eV} /\left(\mathrm{cm}^{2} \mathrm{~s} \mathrm{sr}\right)$ for the highenergy range. This period corresponds very probably to the encounter of Mars with a CIR or a SIR. This CIR/SIR induced an increase of the dynamic pressure and an increase of the energetic particles (Lee et al., 2018, 2017), which can be seen in Figures $3 f$ and 3g, a phenomenon also observed in several other CIR/SIR encounters (Hara et al., 2011; Lee et al., 2017). The measured increase of the SEP ions seems to be associated with the arrival of this solar wind perturbation, and therefore, most probably energetic picked up ions are accelerated by the intense convective electric field (see Figure 3e). Figure $3 \mathrm{c}$ shows that the EUV flux decreased and was smaller than during the other periods.

Figure 8 is a zoom of the fifth period displaying the precipitating ion flux at low (Figure 8a) and high energy (Figure 8b) with the proxy of the convective electric field at MAVEN periapsis (Figure 8c), the reconstructed dynamic pressure at MAVEN periapsis (Figure 8d), and the MSE longitude and latitude position of MAVEN when the penetrating ion flux was measured (Figure 8e). With Figure 8e, it is noticeable that we observe a dichotomy of the intensity of the precipitating ion flux at high energy between the hemisphere $+\mathrm{E}$ and the hemisphere $-\mathrm{E}$, with an intensity of precipitating ion flux stronger in the hemisphere $-\mathrm{E}$ than the hemisphere $+\mathrm{E}$, which is a behavior that has been suggested in Hara, Luhmann, Leblanc, Curry, Seki, et al. (2017). We compared the average precipitating ion flux from 21 to 24 September in the northern $(+E)$ and southern $(-E)$ hemispheres with, on the high-energy range, $1.8 \pm 3.0 \times 10^{8} \mathrm{eV} /\left(\mathrm{cm}^{2} \mathrm{~s} \mathrm{sr} ; 9\right.$ measurements $)$ and $3.6 \pm 1.9 \times 10^{8} \mathrm{eV} /\left(\mathrm{cm}^{2} \mathrm{~s} \mathrm{sr} ; 7\right.$ measurements $)$, respectively. The precipitating ion flux is twice as high in the southern hemisphere with respect to the flux in the northern hemisphere, but due to the relatively limited number of point, this difference remains within the dispersion. Over the whole period, we also note that the evolution of the precipitating ion flux at low and high energy (Figures 8a and 8b) is similar, except for the third and fourth orbits.

During the third orbit, there is a significant precipitating ion flux at high energy. This coincides with the increase of the dynamic pressure few hours before the measurement of the precipitating flux. During the fourth orbit, an important precipitating ion flux at low energy is observed (Figure 8a) but not at high energy which can be explained by the low intensity of the convective electric field. As we can be seen in Figure 8e, the precipitating ion flux is generally greater in the southern hemisphere than in the northern hemisphere. The evolution of the precipitating ion flux during 23 and 24 September (in particular at high energy) can be explained by the solar wind dynamic pressure and the electric field peaking beginning of 24 September. After the first orbit of 24 September, the EUV flux, the SEP ion flux, the solar wind dynamic pressure, and electric field became nominal in association with the precipitating ion flux. 


\section{Discussion and Conclusion}

On 13 September 2017, the most intense solar event observed by MAVEN propagated past Mars (Lee et al., 2018). Such an encounter was a unique opportunity to study the influence of various potential solar drivers on the heavy ion precipitation. As a matter of fact, the whole month of September 2017 displayed interesting and dynamic changes in the solar wind. In this study, we therefore focused on five periods in September 2017, during which the solar conditions significantly changed, as well as the precipitating ion flux intensity.

In a first step, we developed a reconstruction method of the background induced by the SEP event on MAVEN/SWIA spectrometer (Halekas et al., 2015) and used the solar wind proxies constructed by Ma et al. (2018) to estimate the solar wind conditions. Such developments allowed us to investigate the precipitating ion flux responses to the solar energetic events of September 2017. For each period, during which the precipitating ion flux significantly increased, we discussed the different solar drivers that might explain the observed variations of the intensity of the precipitating flux.

We found a clear correlation between the arrival of a CIR/SIR or ICME and the increase of the precipitating ion flux. An increase of the solar wind dynamic pressure seems to lead to an increase of the precipitating ion flux. This result is consistent with studies modeling precipitating ion flux as a function of solar wind parameters (Fang et al., 2013; Wang et al., 2015). However, we also observed periods of low dynamic pressure associated with strong precipitating ion flux (on 6 September, as an example). As a matter of fact, there is no systematic correlation between the intensity of the dynamic pressure and the intensity of the measured precipitating ion flux for this month of observations. Actually, each period described in this work is influenced by various solar parameters (Chaufray et al., 2007; Edberg et al., 2009; Lillis et al., 2015) and most of the time by the concomitant variations of few of them. During one of these periods, we found a significant linear correlation (correlation coefficient of 0.69) between the dynamic pressure and the precipitating ion flux based on 21 measurements, from 17 to 20 September. But, clearly, solar dynamic pressure is not the only driver for ion precipitation during most of September 2017. As discussed in this paper, the measured precipitating ion flux seems also to be correlated with the orientation of the convective electric field (as an example during the period of 21 to 24 September and also shown in Hara, Luhmann, Leblanc, Curry, Seki, et al. (2017)). Another potential solar driver is the SEP flux which seems to favor the increase of the precipitating ion flux. According to Lillis et al. (2015) and Edberg et al. (2009), the EUV and SEP flux can heat and ionize the Martian upper atmosphere, potentially increasing the precipitating ion flux. But it is also necessary to distinguish the high-energy ion planetary and the ion and electron SEPs. For instance, from 21 to 24 September, the measured increase of the ion and electron SEP fluxes is a consequence of the SIR arrival which must have caused a significant acceleration of the planetary picked up ions.

At last, between 7 and 10 September, the EUV flux in the 0.1-7-nm range was from 2 to 3 times higher than the EUV flux during the nominal period, whereas the measured precipitating ion flux did not show any significant increase. During this period, the solar dynamic pressure and convective electric field were also essentially nominal. Therefore, the EUV flux seems not to be a key driver for the precipitating ion flux during this particular period. This conclusion is not in agreement with several past studies (Chaufray et al., 2007; Edberg et al., 2009; Lillis et al., 2015). As an example, according to Chaufray et al. (2007), ion precipitation should be favored by the solar EUV/UV flux. However, this work also highlighted that a higher EUV/UV flux might lead to a higher ionization rate in the Martian corona leading to a higher mass loading of the solar wind acting as a more efficient shielding of the Martian atmosphere. This conclusion regarding the possible role of the EUV flux on the precipitating flux should however be considered with cautious, the one-month sample used in this study being far from statistically significant.

\section{References}

Acuña, M. H., Connerney, J. E. P., Ness, N. F., Lin, R. P., Mitchell, D., Carlson, C. W., McFadden, J., et al. (1999). Global distribution of crustal magnetization discovered by the Mars Global Surveyor MAG/ER experiment. Science, 284(5415), 790-793. https://doi.org/ 10.1126/science.284.5415.790

Barabash, S., Lundin, R., Andersson, H., Brinkfeldt, K., Grigoriev, A., Gunell, H., Holmström, M., et al. (2006). Space Science Reviews, 126(1-4), 113-164. https://doi.org/10.1007/s11214-006-9124-8 
Chassefière, E., \& Leblanc, F. (2004). Mars atmospheric escape and evolution; interaction with the solar wind. Planetary and Space Science, 52(11), 1039-1058. https://doi.org/10.1016/j.pss.2004.07.002

Chaufray, J. Y., Modolo, R., Leblanc, F., Chanteur, G., Johnson, R. E., \& Luhmann, J. G. (2007). Mars solar wind interaction: Formation of the Martian corona and atmospheric loss to space. Journal of Geophysical Research, 112, E09009. https://doi.org/10.1029/ 2007JE002915

Connerney, J. E. P., Espley, J., Lawton, P., Murphy, S., Odom, J., Oliversen, R., \& Sheppard, D. (2015). The MAVEN magnetic field investigation. Space Science Reviews, 195(1-4), 257-291. https://doi.org/10.1007/s11214-015-0169-4

Curry, S. M., Luhmann, J. G., Ma, Y. J., Dong, C. F., Brain, D., Leblanc, F., Modolo, R., et al. (2015). Response of Mars 0+ pickup ions to the 8 March 2015 ICME: Inferences from MAVEN data-based models. Geophysical Research Letters, 42, 9095-9102. https://doi.org/10.1002/ 2015GL06304

Delory, G. T., Luhmann, J. G., Brain, D., Lillis, R. J., Mitchell, D. L., Mewaldt, R. A., \& Falkenberg, T. V. (2012). Energetic particles detected by the electron reflectometer instrument on the Mars global surveyor 1999-2006. Space Weather, 10, S06003. https://doi.org/10.1029/ 2012SW000781

Edberg, N., Brain, D. A., Lester, M., Cowley, S. W. H., Modolo, R., Franz, M., \& Barabash, S. (2009). Plasma boundary variability at Mars as observed by Mars global surveyor and Mars express. Annales de Geophysique, 27(9), 3537-3550. https://doi.org/10.5194/angeo-273537-2009

Eparvier, F. G., Chamberlin, P. C., Woods, T. N., \& Thiemann, E. M. B. (2015). The solar extreme ultraviolet monitor for MAVEN. Space Science Reviews, 195(1-4), 293-301. https://doi.org/10.1007/s11214-015-0195-2

Fang, X., Bougher, S. W., Johnson, R. E., Luhmann, J. G., Ma, Y., Wang, Y.-C., \& Liemohn, M. W. (2013). The importance of pickup oxygen ion precipitation to the Mars upper atmosphere under extreme solar wind conditions. Geophysical Research Letters, 40, $1922-1927$. https://doi.org/10.1002/grl.50415

Frahm, R. A., Sharber, J. R., Winningham, J. D., Elliott, H. A., Howard, T. A., DeForest, C. E., Odstrĉil, D., et al. (2013). Solar energetic particle arrival at Mars due to the 27 January 2012 solar storm. AIP Conference Proceedings, 1539, 394. https://doi.org/10.1063/1.4811066

Guo, J., Dumbović, M., Wimmer-Schweingruber, R. F., Temmer, M., Lohf, H., Wang, Y., Veronig, A., et al. (2018). Modeling the evolution and propagation of September 2017 CMEs and SEPs arriving at Mars constrained by remote sensing and in situ measurement. Space Weather, 16, 1156-1169. https://doi.org/10.1029/2018SW001973

Halekas, J. S., Taylor, E. R., Dalton, G., Johnson, G., Curtis, D. W., McFadden, J. P., Mitchell, D. L., et al. (2015). The solar wind ion analyzer for MAVEN. Space Science Reviews, 195(1-4), 125-151. https://doi.org/10.1007/s11214-013-0029-z

Hara, T., Luhmann, J. G., Leblanc, F., Curry, S. M., Halekas, J. S., Seki, K., Brain, D. A., et al. (2017). Evidence for crustal magnetic field control of ions precipitating into the upper atmosphere of Mars. Journal of Geophysical Research: Space Physics, 123, $8572-8586$. https://doi.org/10.1029/2017JA024798

Hara, T., Luhmann, J. G., Leblanc, F., Curry, S. M., Seki, K., Brain, D. A., Halekas, J. S., et al. (2017). MAVEN observations on a hemispheric asymmetry of precipitating ions toward the Martian upper atmosphere according to the upstream solar wind electric field. Journal of Geophysical Research: Space Physics, 122, 1083-1101. https://doi.org/10.1002/2016JA023348

Hara, T., Seki, K., Futaana, Y., Yamauchi, M., Yagi, M., Matsumoto, Y., Tokumaru, M., et al. (2011). Heavy-ion flux enhancement in the vicinity of the Martian ionosphere during CIR passage: Mars express ASPERA-3 observations. Journal of Geophysical Research, 116, A02309. https://doi.org/10.1029/2010JA015778

Jakosky, B. M., Lin, R. P., Grebowsky, J. M., Luhmann, J. G., Mitchell, D. F., Beutelschies, G., Priser, T., et al. (2015). The Mars Atmosphere and Volatile EvolutioN (MAVEN) mission. Space Science Reviews, 195(1-4), 3. https://doi.org/10.1007/s11214-015-0139-x-48.

Johnson, R. E. (1994). Plasma-induced sputtering of an atmosphere. Space Science Reviews, 69(3-4), 215-253. https://doi.org/10.1007/ BF02101697

Larson, D. E., Lillis, R. J., Lee, C. O., Dunn, P. A., Hatch, K., Robinson, M., Glaser, D., et al. (2015). The MAVEN solar energetic particle investigation. Space Science Reviews, 195(1-4), 153-172. https://doi.org/10.1007/s11214-015-0218-z

Leblanc, F., Chaufray, J. Y., Modolo, R., Leclercq, L., Curry, S., Luhmann, J., Lillis, R., et al. (2017). On the origins of Mars' exospheric nonthermal oxygen component as observed by MAVEN and modeled by HELIOSARES. Journal of Geophysical Research:Planets, 122, 2401-2428. https://doi.org/10.1002/2017JE005336

Leblanc, F., \& Johnson, R. E. (2001). Sputtering of the Martian atmosphere by solar wind pick-up ions. Planetary and Space Science, 49(6), 645-656. https://doi.org/10.1016/S0032-0633(01)00003-4

Leblanc, F., \& Johnson, R. E. (2002). Role of molecules in pick-up ion sputtering of the Martian atmosphere. Journal of Geophysical Research, 107(E2), 5010. https://doi.org/10.1029/2000JE001473

Leblanc, F., Martinez, A., Chaufray, J. Y., Modolo, R., Hara, T., \& Luhmann, J. (2018). On Mars' atmospheric sputtering after MAVEN first Martian year of measurements. Geophysical Research Letters, 45, 4685-4691. https://doi.org/10.1002/2018GL077199

Leblanc, F., Modolo, R., \& al. (2015). Mars heavy ion precipitating flux as measured by Mars atmosphere and volatile EvolutioN. Geophysical Research Letters, 42, 9135-9141. https://doi.org/10.1002/2015GL066170

Lee, C. O., Hara, T., Halekas, J. S., Thiemann, E., Chamberlin, P., Eparvier, F., Lillis, R. J., et al. (2017). MAVEN observations of the solar cycle 24 space weather conditions at Mars. Journal of Geophysical Research: Space Physics, 122, 2768-2794. https://doi.org/10.1002/ 2016JA023495

Lee, C. O., Jakosky, B. M., Luhmann, J. G., Brain, D. A., Mays, M. L., Hassler, D. M., Holmström, M., et al. (2018). Observations and impacts of the 10 September 2017 solar events at Mars: An overview and synthesis of the initial results. Geophysical Research Letters, 45 , 8871-8885. https://doi.org/10.1029/2018GL079162

Lillis, R. J., Brain, D. A., Bougher, S. W., Leblanc, F., Luhmann, J. G., Jakosky, B. M., Modolo, R., et al. (2015). Characterizing atmospheric Escape from Mars today and through time, with MAVEN. Space Science Reviews, 195(1-4), 357-422. https://doi.org/10.1007/s11214-0150165-8

Luhmann, J. G., Johnson, R. E., \& Zhang, M. H. G. (1992). Evolutionary impact of sputtering of the Martian atmosphere by $\mathrm{O}^{+}$pickup ions. Geophysical Research Letters, 19, 2151-2154. https://doi.org/10.1029/92GL02485

Luhmann, J. G., \& Kozyra, J. U. (1991). Dayside pickup oxygen ion precipitation at Venus and Mars: Spatial distributions, energy deposition and consequences. Journal of Geophysical Research, 96, 5457-5467. https://doi.org/10.1029/90JA01753

Ma, Y., Fang, X., Halekas, J. S., Xu, S., Russell, C. T., Luhmann, J. G., Nagy, A. F., et al. (2018). The impact and solar wind proxy of the 2017 September ICME event at Mars. Geophysical Research Letters, 45, 7248-7256. https://doi.org/10.1029/2018GL077707

McFadden, J. P., Kortmann, O., Curtis, D., Dalton, G., Johnson, G., Abiad, R., Sterling, R., et al. (2015). MAVEN SupraThermal and thermal ion composition (STATIC) instrument. Space Science Reviews, 195(1-4), 199-256. https://doi.org/10.1007/s11214-015-0175-6 
Modolo, R., Hess, S., Mancini, M., Leblanc, F., Chaufray, J. Y., Brain, D., Leclercq, L., et al. (2016). Mars-solar wind interaction: LatHyS, an improved parallel 3-D multispecies hybrid model. Journal of Geophysical Research: Space Physics, 121, 6378-6399. https://doi.org/ 10.1002/2015JA022324

Rahmati, A., Larson, D. E., Cravens, T. E., Lillis, R. J., Dunn, P. A., Halekas, J. S., Connerney, J. E., et al. (2015). MAVEN insights into oxygen pickup ions at Mars. Geophysical Research Letters, 42, 8870-8876. https://doi.org/10.1002/2015GL065262

Rahmati, A., Larson, D. E., Cravens, T. E., Lillis, R. J., Halekas, J. S., McFadden, J. P., Dunn, P. A., et al. (2017). MAVEN measured oxygen and hydrogen pickup ions: Probing the Martian exosphere and neutral escape. Journal of Geophysical Research: Space Physics, 122, 3689-3706. https://doi.org/10.1002/2016JA023371

Ramstad, R., Holmström, M., Futaana, Y., Lee, C. O., Rahmati, A., Dunn, P., Lillis, R. J., et al. (2018). The September 2017 SEP event in context with the current solar cycle: Mars express ASPERA-3/IMA and MAVEN/SEP observations. Geophysical Research Letters, 45, 7306-7311. https://doi.org/10.1029/2018GL077842

Romanelli, N., Modolo, R., Leblanc, F., Chaufray, J.-Y., Martinez, A., Ma, Y., Lee, C., et al. (2018). Responses of the Martian magnetosphere to an interplanetary coronal mass ejection: MAVEN observations and LatHyS results: Response of MARS to a CME. Geophysical Research Letters, 45, 7891-7900. https://doi.org/10.1029/2018GL077714

Wang, Y.-C., Luhmann, J. G., Fang, X., Leblanc, F., Johnson, R. E., Ma, Y., \& Ip, W.-H. (2015). Statistical studies on Mars atmospheric sputtering by precipitating pickup $\mathrm{O}^{+}$: Preparation for the MAVEN mission. Journal of Geophysical Research:Planets, 120, 34-50. https://doi.org/10.1002/2014JE004660

Xu, S., Fang, X., Mitchell, D. L., Ma, Y., Luhmann, J. G., DiBraccio, G. A., Weber, T., et al. (2018). Investigation of Martian magnetic topology response to 2017 September ICME. Geophysical Research Letters, 45, 7337-7346. https://doi.org/10.1029/2018GL077708 